\title{
Medicine Drugs Circuit: Analyze Performance with Simulation Models
}

\author{
F. OUZAYD \\ Ph.D student \\ National Higher School of \\ Electricity and Mechanics \\ (ENSEM) \\ Hassan II University- \\ Casablanca-Morocco,
}

\author{
J. SAADI \\ Professor \\ National Higher School of \\ Electricity and Mechanics \\ (ENSEM) \\ Hassan II University, \\ Casablanca, Morocco,
}

\author{
J. BENHRA \\ Professor \\ National Higher School of \\ Electricity and Mechanics \\ (ENSEM) \\ Hassan II University- \\ Casablanca-Morocco,
}

\author{
A. ZAKARI \\ Professor \\ Université de Lorrainne \\ LITA Metz - France \\ (Laboratoire \\ d'Informatique Théorique \\ et Appliquée)
}

\begin{abstract}
Hospital mission is to insure patient safety and quality of care associated with any medical processor. Among to provide performance hospitals processors is medicine drugs circuit. Modeling and simulating is an excellent tool that allows understanding this complexity and analyzing performances. In this paper, we propose a simulation models with Colored Petri Net (CPN) of Medicine Drugs Chain. Being based on these models, we present a simulating Queue Patient model with CPN Tools which will allow some statistics measures.
\end{abstract}

\section{Keywords}

Hospital, medicine drugs chain, Colored Petri Net, performance.

\section{INTRODUCTION}

The health sector in Morocco is largely depending on public demand. In fact, $85 \%$ of the medical equipment is issued by the Ministry of Public Health, the Ministry of Defense, the Security Fund (SF) and University Hospital Center (UHC). The majority of the demand coming mainly the Moroccan Ministry of Health (MMH) with a budget of nearly 10.5 billion Dirham in 2010 (against 9.8 billion Dirham's in 2009), representing $5.5 \%$ of budget the state and $1.3 \%$ of global public expenses. However, the sector has a huge need in medical staff. MMH lists 10933 physicians (generalists, plasticize and other specialties) and nurses, there are currently about 8.5 nurses per 10000 inhabitants or approximately one nurse for every 1,134 inhabitants in the public area [1]. In addition, Morocco has a low hospital capacity. Taking into account that private hospitals have a capacity of about 6900 beds, and the total capacity of public institutions ambulatory is about 3700 beds, and hospitals have about 26,000 beds, the supply country overall amount to almost 37000 beds. In reporting this figure to the Moroccan population yields a ratio of about 11 beds for 10000 inhabitants. This figure puts Morocco behind Algeria (17 beds/10 000), Tunisia ( 24 beds/10 000) but ahead of the Sultanate of Oman and Qatar (with 9 beds per 10000 inhabitants) [2]. In this context, the health sector is in rough conditions binding by weak financial and material resources. This requires urgent intervention in the aim to readdress these constraints and improve a service care to patients. In our case, we focus to important role of hospital supply and particularly medicine drugs chain. The medicine drugs chain is consisting of one hand of the medication chain whose goal is clinical care of patients and other hand hospital supply chain that is committed to insure the availability drugs to patients. The

failing at the level of the process can generate of the undesirable effects and sometimes died of the patient. The safety of patient requires a safety clinical chain and an optimized hospital supply chain. For that, more authors resort generally to modeling and simulation as [3], [4], [5]-[6] and [7]. That's because it's an excellent approach to provide the usual and efficient tool for the scheduling of capacities and the improvement of the efficiency. In this paper, in the same way, we propose simulation models of medicine drugs chain in Moroccan healthcare system with CPN Tools. The remainder of the paper is organized as follows. Section 2, we explain medicine drugs chain. Section 3, we propose several models of medicine drugs chain with Colored Petri Net and finally we simulate by CPN Tools plate-form.

\section{MEDICINE DRUGS CIRCUIT}

Hospital mission's is to insure patient safety and quality of care associated with any medical processor. Among to provide performance hospitals processors is medicine drugs chain. Thought a several definitions proposed by authors in last decade, we present two terms: drugs circuit and supply medicine drugs chain. These expressions give a same meaning. Drug circuit in hospital includes three main steps: prescribing, dispensing and administration. It's consisting of one hand of the clinical chain whose goal is clinical care of patients and other hand hospital supply chain that is committed to assure the availability drugs to patients. The Direction for Hospitalization and Organization of Care [8] in France define a drugs circuit to distinguish tow flows: material flow and informational flow. The materiel flow includes controlled drug delivery to the pharmacy, to storage, preparing and delivery of drugs in healthcare units, administration of drugs to patients and return management. The second is consisting of medication management which affects more order and transmitting information relating to the drug between members of the healthcare team. For Aloui [9] drugs circuit is one hand a medical care system and the other hand transverse to structure in several health facilities. Therefore, Schmitt, propose other definition of drugs circuit to extending this processes of care of patient hospitalized in a health facility and a resident of a facility or outpatient social [10].

Generally, drugs circuit consists of two channels: first chain is responsible for the clinical management of patient with the process of prescribing the order of drug distribution and administration and second chain is the supply chain. It is a complex system whose mission is to ensure patient safety and to provide whatever service configuration applicants or the pressure of the environment; the right drug to the right patient in good condition at the right time and [11] added another goal by attending on controlling costs for institutions. Thus, safety and performances of medicine drugs are conditions at all hospital supply chain. Therefore, modeling and simulation approaches seem excellent tools for resolving such challenges. In Moroccan Hospital system and particularly Hospital University Center, a medicine drugs chain consist on 
administration patient, prescription, global dispensation and finally administration, the supply system is centralized at the Supply Division. His role is to call of suppliers and dispatch the drugs toward hospital pharmacy. Healthcare Units reorder by adopting the global dispensing system.

\section{LITERATURE REVIEW: MODELING FRAMEWORK AND OPTIMIZATION TECHNIQUES OF DRUGS CIRCUIT}

In the last decades, more and more of researchers, focus in hospital systems problematic and particularly drugs circuit. In this paper, we present several works treating this problematic since 2006.

Hassan [12] sought in his study to organize the downstream pharmaceutical chain and consumable products optimization. For that, the author has made a model of strategic and tactical levels by SCOR (Supply Chain Operations Reference) and simulation of operational level by ARIS platform. In the other work, Saber [13] proposes a framework to share the methodology for risk management organizations such as hospitals. The approach is based on the systemic paradigm and implements a system engineering approach based on three levels of modeling. First, for the modeling phase, that is to say, the abstraction of the system in several meta-models, the author has used tools and languages such as GME (Generic Modeling Environment), UEML (Unified Enterprise Modelling Language) and eFFBD (Enhanced Functional Flow Block Diagram). Then, through a phase of analysis by finding a satisfactory level of confidence in the models for verification and validation of partial models, the author referred to tools such as XME2CogXML and Cogitant. Finally, in specification step, the objective is to analyze various risks that may affect for example the circuit drugs. The objective of this work is achieved by the development of approach for the engineering systems for representing and analyzing complex sociotechnical systems such as the drugs circuit. In same study, Sienou [14] proposed a methodological framework for the integrated management of risks and business processes. The author proposes the meta-models made by the ARIS tool whose objective is to develop a method of unifying process engineering business processes and risk by applying this approach to the drugs circuit. However, Baboli [15], to treat logistic problem, provider mathematical models to compare the performance local and global pharmaceutical downstream supply chain in the structure and decision-making is centralized or not. To simulate these models, experimental results show that the centralized approach is more efficient from a global perspective, but a mechanism for distribution of earnings is necessary locally because some links are losers. To trait problem of planning and allocation of resources in a hospital pharmacy and especially to generate a feasible schedule for preparers, Cresty [16] try to solve this NP-hard problem by new meta-heuristic that allow to automatic generation of schedules for the staff of hospital pharmacy. The proposed method is based on a variation of the algorithm of ant colony optimization for task scheduling and specific heuristic for assigning tasks to resources based on their skills. Several numerical tests are proposed to demonstrate the effectiveness of the method. Huet [17] aims to automate dispensing drugs system in hospital. The authors have developed a model combining knowledge-Holon Manufacturing System and constituting a UML tool for the decision for the organization of the drugs circuit. This author [18], in extend study, offers a methodology for re-engineering the product control of manufacturing systems implement this approach to drug supply in a hospital. The author has developed models in AutoMod software. Whose goal is to reduce drug iatrogenic risks, the author proposes a methodology called RMHMS (Reengineering Methodology of Holonic Manufacturing System) to model, simulate and deploy reorganization of drugs circuit in university hospital center Clermont Ferrand.

Along this literature review, in recent year's treating drug circuit problem, we found that most of the studies apply a modeling approach. We remark also that these studies focus on the mathematical modeling by adopting optimization algorithms in the case of meta-heuristics, heuristic, exact mathematical models or graphical modeling. Indeed, because objective of our work is proposed is modeling and simulation the drugs circuit in Moroccan system, we interest more of graphical modeling. In fact, we find that most of these studies are interested to propos of methodological frameworks with application example are the drugs circuit, as the work of, [18] [13] and [15] including in some cases risk propriety. We remark also that the work presented by [17] and not the system as a whole. Moreover, our model is a global model that can analyze each step of drug circuit since the admission of the patient until the patient is discharged with the analysis of different points: waiting time for patients in tick box, the waiting time for patients in the care rooms, the time of utilization of resources like doctors, registers, nurses and tick boxes. It is open models for analyzing the performance by simulating various existences scenarios to propose improvement areas. Furthermore, the important of our models that reflects the real situation in Morocco drug circuit, something that was never treated, and offers lines of analysis with experimental results.

\section{MODELING MEDICINE DRUGS CHAIN}

4.1. Casablanca university hospital center

Based on 2010 report, the Casablanca university hospital center registered 224 beds as capacity, 28509 admissions, 77195 days of hospitalization, 11500 surgical interventions, 28396 emergency consultations, 75986 external's consultations and 22770 radiology tests. The center contains nine healthcare units: Reanimation Anesthesia, Clinic Hematology, Maxillafacial Chirurgic, O.R.L, Adult Ophthalmology, Pediatric Ophthalmology, Pneumology, Radiology and Emergency. All of these units seen supply to central pharmacy. All these healthcare units can monthly procure of drugs to hospitable pharmacy. A supply division localized in hospital insures quality drugs purchasing to nationals and internationals pharmacists suppliers.

\subsection{Colored Petri Net}

To modeling a medicine drugs circuit, we use Colored Petri Net (CPN). CPN offers a modeling framework that is perfectly for distributed and concurrent processes with both synchronous and asynchronous communication [19]. In addition, they are useful in modeling both non deterministic and stochastic processes as well.

We introduce for brief presentation of Colored Petri Net theory, developed by [20].

CPN is a tuple $(\Sigma, \mathrm{P}, \mathrm{T}, \mathrm{A}, \mathrm{N}, \mathrm{C}, \mathrm{G}, \mathrm{E}, \mathrm{I})$ satisfying the following requirements:

(1) $\Sigma$ is a finite set of non-empty types, also called color sets.

(2) $\mathrm{P}$ is a finite set of places.

(3) $\mathrm{T}$ is a finite set of transitions.

(4) $\mathrm{A}$ is a finite set of arcs such that: $\mathrm{P} \cap \mathrm{T}=\mathrm{P} \cap \mathrm{A}=\mathrm{T} \cap \mathrm{A}$ $=\varnothing$. 
(5) $\mathrm{N}$ is a node function. It is defined from $\mathrm{A}$ into $\mathrm{P} x \mathrm{~T} \cup \mathrm{T}$ $\mathrm{x} P$.

(6) $\mathrm{C}$ is a color function. It is defined from $\mathrm{P}$ into $\Sigma$.

(7) $G$ is a guard function. It is defined from $T$ into expressions such that: $\forall \mathrm{t} \in \mathrm{T}$ : [Type $(\mathrm{G}(\mathrm{t}))=\mathrm{B} \wedge$ Type $(\operatorname{Var}(\mathrm{G}(\mathrm{t}))) \subseteq \mathrm{S}]$.

(8) $\mathrm{E}$ is an arc expression function. It is defined from $\mathrm{A}$ into expressions such that: $\forall \mathrm{a} \in \mathrm{A}:[\operatorname{Type}(\mathrm{E}(\mathrm{a}))=\mathrm{C}(\mathrm{p}) \mathrm{MS} \wedge$ Type $(\operatorname{Var}(E(a))) \subseteq S]$ where $p$ is the place of $N(a)$.

(9) $\mathrm{I}$ is an initialization function. It is defined from $\mathrm{P}$ into closed expressions such that: $\forall \mathrm{p} \in \mathrm{P}$ : [Type $(\mathrm{I}(\mathrm{p}))=\mathrm{C}(\mathrm{p}) \mathrm{MS}$ ]

CPN is tool and framework that allow design, specification, validation, and verification of systems [21].

In this work, we can be subdivided in three sub models which regrouping in hierarchic model (fig.1). We have a queue patient in waiting room (sub model 1), Registration patient (sub model 2) and medicine drugs circuit (prescription-dispensationadministration and logistic system) (sub model 3).

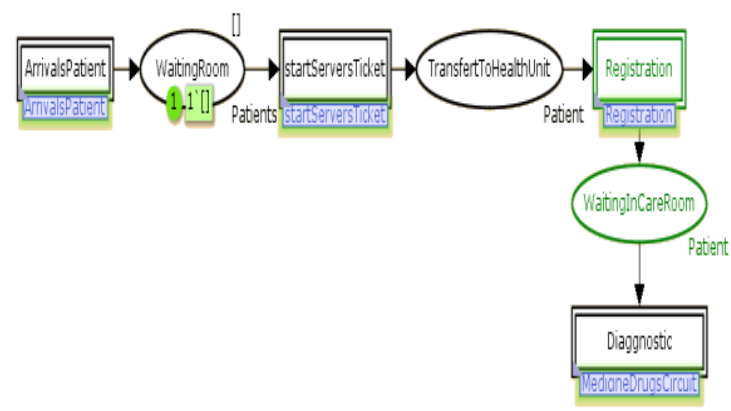

Figure 1: Mean model

\subsection{Queue Patient sub model}

This means that tokens residing on the two places INIT and NextPatient will have an integer as their token color. The color set $\mathrm{U}$ is used to model the time of arrival patient. The place WaitingRoom has the color set PATIENTS defined to be the set of list patient. The color set PATIENTS is used to model the payload of patients to be treated. The color set Patient models a patient as a record consisting of two fields. The field named patientType determines the type of the patient since it has type PatientType. The AT field is of type INT, and it is used to store the arrival time of the patient. The color set TicketboxxPatient a product color set that is used to present the ticket box when it is busy processing for procuring a ticket (server patient). The function expTime is used to generate integer values that are approximately exponentially distributed with a mean value determined by the parameter mean. The function uses the exponential random distribution. We use this distribution because we can't determine the inter-arrival time for reception patient in waiting room. The place WaitingRoom models the queue of patients. There is a single token on the place in the initial marking of the net, and there is be a single token on the place in every reachable marking. The single token on the place represents the queue of patients. In the initial marking the list is empty. Patients are added to the queue in the Arrivals sub-module, and patients are removed from the WaitingRoom. In waiting room, the patient takes his ticket which contains the number, the affected healthcare unit (each patient assign for one category on unit healthcare that he must to affect), time for admission.
The places Free and Busy are used to represent the status of the ticket box. A token on place Free indicates that the ticket box is not processing to serve a patient. There is a single token on the place Free in the initial marking. A token on place Busy indicates that the server is processing to procure admission ticket, and the value of the token indicates which patient is being processed. The initial marking of Busy is empty. The ticket box can start processing to procure a ticket (transition startServerTicket), if the ticket box is Free and if there is at least one patient in the queue of patients (patient::patients on the arc from place WaitingRoom).

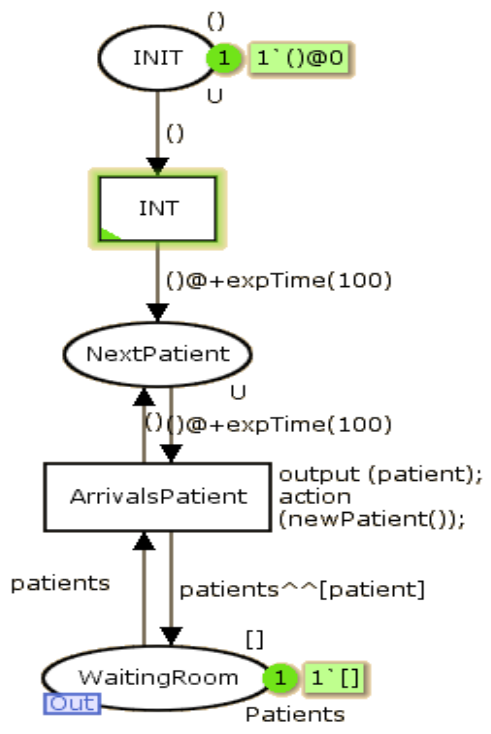

Figure 2: ArrivalsPatient module

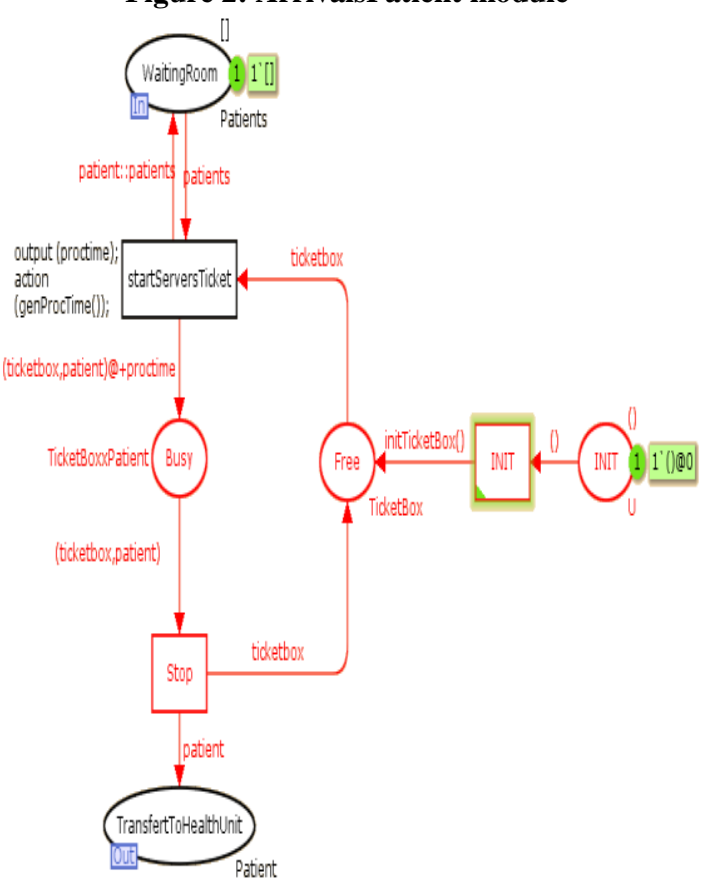

Figure 3: StartServersTicket module

\subsection{Registration sub model}

Patient transfers to healthcare unit based on the admission ticket, wait in the queue for registration. In administration procedure, a nurse registers all patient information and creates 
a new medical file, if the patient comes for first time. The medical file contains: name, age, incoming date, and disease type, etc. We consider a MedicalFile place modeled two states: first time or existing medical file. The patient will be added to the place Queue by firing the arrivals patients To Healthcare Unit transition. The place Queue models the queue of patients. There is a single token on the place in the initial marking of the net, and there is be a single token on the place in every reachable marking.

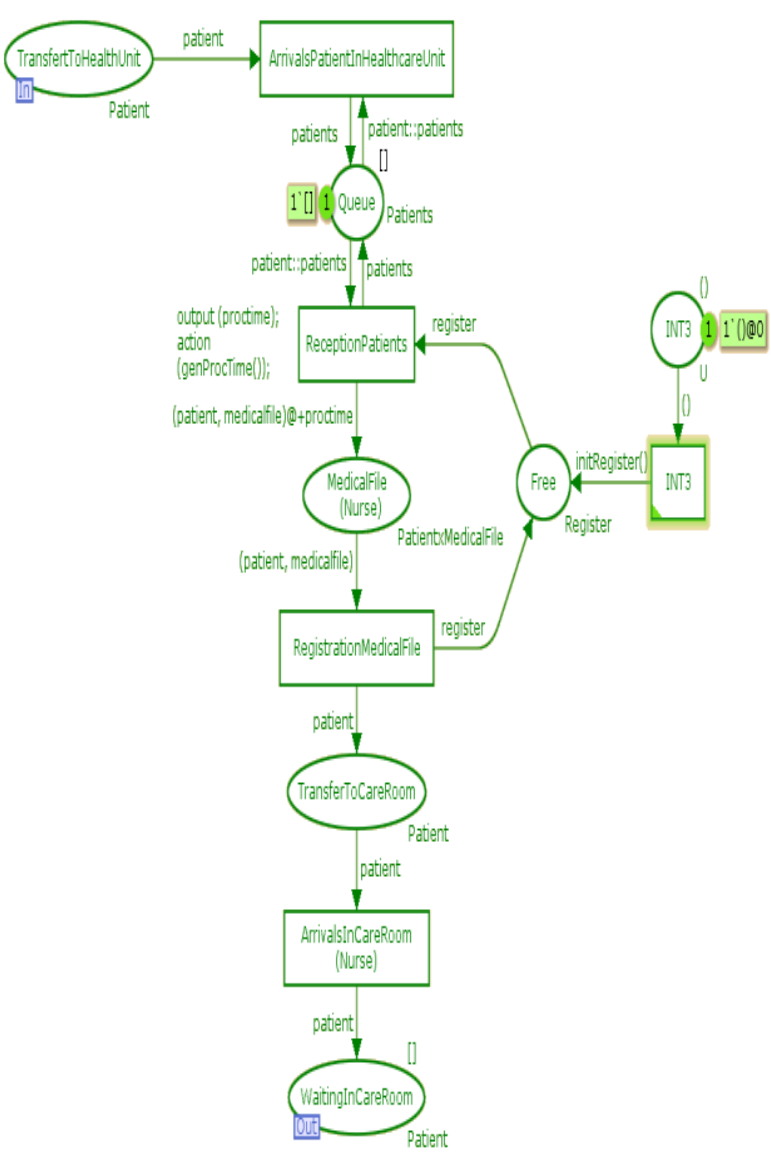

Figure 4: Registration patient sub model

\subsection{Medicine drugs circuit sub model}

Modeling medicine drugs circuit start by prescription step. In center, a doctor makes a pathology diagnostic, for distinguishing two type of prescription (like a patient type). A patient can have a long stay prescription (LS) or consultation prescription (CP).

For global dispensation, nurse evaluates the "fixed quantity of drugs"; if the quantity in healthcare unit pharmacy is less than 2000 units, send a command to the central pharmacy. The place DrugsToRoom presents the packet of drugs sending to room for administration the drugs for patient.

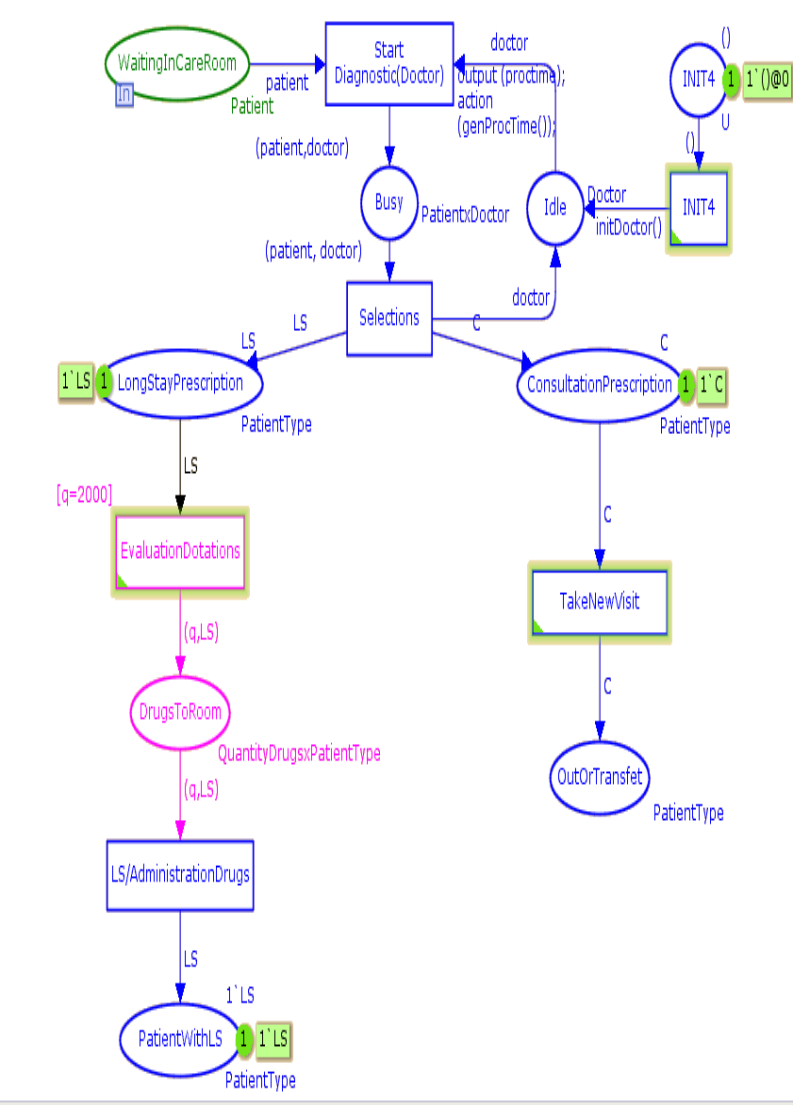

Figure 5: Medicine drugs circuit sub model

\section{Simulation AND RESUlts}

The evaluation presented in this work focuses on control performance by measuring total time to take care of patient and queue length patient.

\subsection{Simulation declarations}

With this model, we can evaluate several scenarios in medicine drugs chain, but in our case, we focused to measure total time total time to take care of patient and length queue in ticket box.

We use two declarations: simulation and monitoring declarations. For simulation, we fix a following example of declaration:

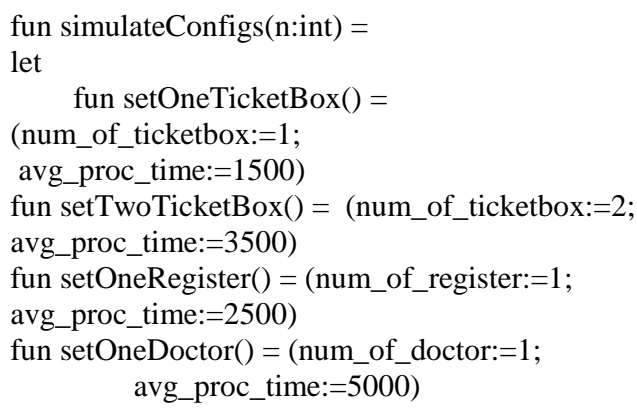

We use an example of global monitoring declarations:

- $\quad$ globref longdelaytime $=2000$;

- $\quad$ globref fileid $=($ NONE $:$ TextIO.outstream option $)$;

- $\quad$ fun initfile ()$=$ let

$$
\text { val filename }=\text { OS.Path.concat }
$$

(Output.getSimOutputDir(), 


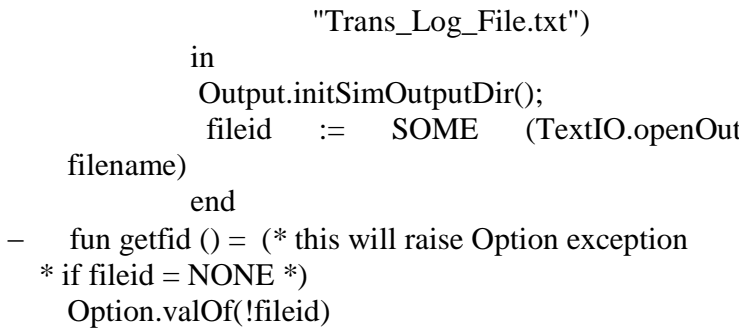

And for evaluate performance of chain, we use three monitors: Queue patient (delay and length), waiting in care room patient and register and doctor utilization.

Monitors
QueuePatient module
Queue Delay
Queue Lenght
TicketBox Utilization
Registration module
Doctor module
Queue3 Delay
Doctor utilization
Marking_size_MedicineDrugsCircuit'Busy_1
Doctor utilisation
ProcessorTime3
Doctor utilization estimate by proctime
Doctor utilization by proctime
QueuePatient

Figure 6: Monitoring declaration of models

\subsection{Analyze a performance}

The simulation with 6 sub runs, covering 2000 steps per run and in the model was set to $1 / 100$ of a minute. The queuing strategy for the queue is first-in-first-out (FIFO). If the ticket box is idle when a patient arrives, the patient passes immediately through the (empty) queue, and the ticket box begins to process admission ticket for patient. When the ticket box finishes processing admission ticket, it becomes idle. If the ticket box is idle and there are patients waiting in the queue, then the ticket box will immediately begin to process admission ticket at the head of the queue. We suppose available quantity of drugs in healthcare unit pharmacy.

We present in table 1 statistics results that generated by CPN Tools.

CPN Tools Simulation Performance Report

Net:

/cygdrive/D/Paper2012/Simulation1/MedecineDrugsCircuitIn MoroccoGlobal.cpn

Note that these statistics have been calculated for data that is not necessarily independent or identically distributed.

\begin{tabular}{|c|c|c|c|c|}
\hline \multicolumn{5}{|c|}{ Statistics results } \\
\hline Name & Sum & Avrg & Min & Max \\
\hline DoctorUtilisation & 107 & 0.50 & 0 & 1 \\
\hline $\begin{array}{l}\text { DoctorUtilization } \\
\text { EstimateByProctime }\end{array}$ & $\sim 47.483$ & $\sim 0.323$ & $\sim 1.00$ & 1.63 \\
\hline ProcessorTime3 & 43354 & 405.18 & 2.00 & 1758 \\
\hline ProcessorTime2 & 35330 & 327.13 & 0.000 & 1912 \\
\hline QueuePatientIntInf & 51176 & 302.82 & 0 & 1340 \\
\hline $\begin{array}{l}\text { QueuePatient } \\
\text { NoInitNoStop }\end{array}$ & 617 & 1.8041 & 0 & 9 \\
\hline $\begin{array}{l}\text { QueuePatientReal } \\
\text { Delay }\end{array}$ & 51176 & 302.82 & 0.000 & 1340 \\
\hline QueueIntInf & 131386 & 1216.6 & 28 & 8694 \\
\hline QueueLenght & 681 & 1.78 & 0 & 9 \\
\hline QueueLenghtNoInit & 681 & 1.78 & 0 & 9 \\
\hline QueueLenghtNoStop & 617 & 1.80 & 0 & 9 \\
\hline $\begin{array}{l}\text { QueueLenght } \\
\text { Advanced }\end{array}$ & 653 & 1.84 & 0 & 9 \\
\hline QueueRealdelay & 131386 & 1216.5 & 28.00 & 8694 \\
\hline $\begin{array}{l}\text { RegisterUtilisationBy } \\
\text { Proctime }\end{array}$ & 58.42 & 1.46 & 1.09 & 1.61 \\
\hline RegisterUtilization & 108 & 0.42 & 0 & 1 \\
\hline $\begin{array}{l}\text { RegisterUtilization } \\
\text { EstimateByProcTime }\end{array}$ & 59.52 & 1.49 & 1.15 & 1.63 \\
\hline TicketBoxUtilization & 169 & 0.50 & 0 & 1 \\
\hline $\begin{array}{l}\text { TicketBoxUtilization } \\
\text { Advanced }\end{array}$ & 231 & 0.89 & 0 & 2 \\
\hline $\begin{array}{l}\text { TicketBoxUtilization } \\
\text { ByProctime }\end{array}$ & 59.40 & 1.49 & 1.15 & 1.63 \\
\hline $\begin{array}{l}\text { TicketBoxutilization } \\
\text { estimatebyProcTime }\end{array}$ & 59.52 & 1.48 & 1.15 & 1.63 \\
\hline TotalProcessingTime & 56240 & 332.78 & 1.00 & 1969 \\
\hline $\begin{array}{l}\text { WaitingInCareRoom } \\
\text { IntInf }\end{array}$ & 164225 & 1534.82 & 123 & 9130 \\
\hline $\begin{array}{l}\text { WaitingInCareRoom } \\
\text { RealDelay }\end{array}$ & 164225 & 1534.82 & 123 & 9130 \\
\hline
\end{tabular}

Simulation steps executed: 2000

Model time: 34589

Generated: Fri Aug 17 16:11:22 2012

Table 1: Statistics results generating by CPN Tools 
In this figure, we take an example of simulation scenario for week one that the leads parameters are:

- Fixed quantity drugs: 250;

- Register number: 1;

- Doctor number in care room: 1 ;

- Ticket boxes number: 2;

- Average processes time: 3.60 minutes;

- Inter-arrivals time: 2.00 minutes;

We present in table 2 the total time to take care of patient that amount is 29.03 minutes. This time combining: diagnostic time (ProcessorTime3: 4.05 minutes), registration time (ProcessorTime2: 3.27 minutes) and reception time (ProcessorTime1: 3.32 minutes).

\begin{tabular}{|l|c|}
\cline { 2 - 2 } \multicolumn{1}{c|}{} & Week 1 \\
\hline ProcessorTime3 & 405,17757 \\
\hline ProcessorTime2 & 327,12963 \\
\hline ProcessingTime1 & 332,781065 \\
\hline Total & $\mathbf{1 0 6 5 , 0 8 8 2 6 5}$ \\
\hline QueuePatientRealDelay & 302,816568 \\
\hline WaitingInCareRoomRealDelay & 1534,813084 \\
\hline Total time to take car patient & $\mathbf{2 9 0 2 , 7 1 7 9 1 7}$ \\
\hline
\end{tabular}

Table 2: Means statistics results for simulation on week 1

With model, we evaluate a total time to take care of patient that is composing of: queue patient in ticket box delay, registration time, waiting in care room delay and prescription time.

We compute of this time for 6 weeks. In figure 7, we remark that time is increased in 2 and 3 week and stabilize in 4, 5 and 6 week. For figure 8, we observe of number of patients in waiting room to take admission ticket. We remark that queue length increase for 1.78 patients to 62.25 patients in week 2 and week 3 . In fact, this variation was resulted organizational problems in hospital and introduces a new medical system.

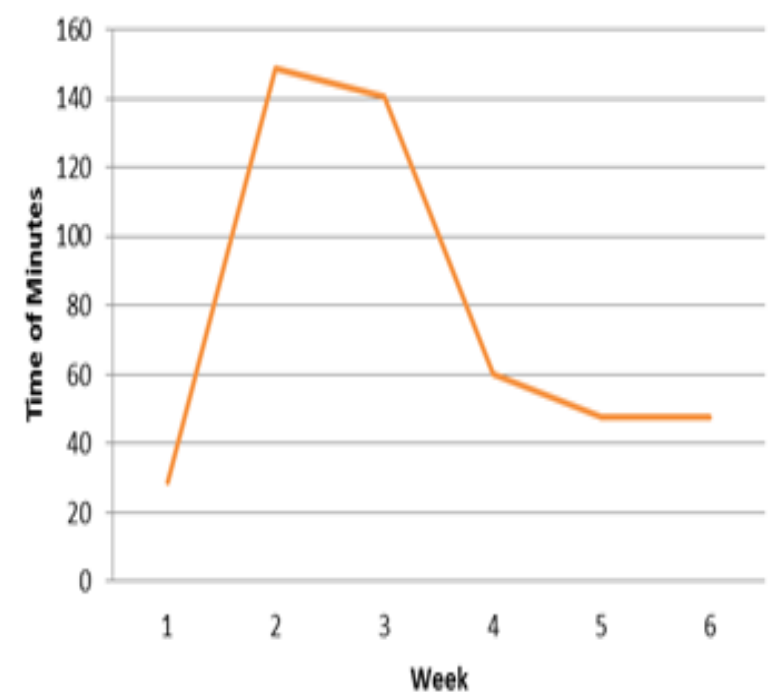

Figure 7: Total time to take care of patient

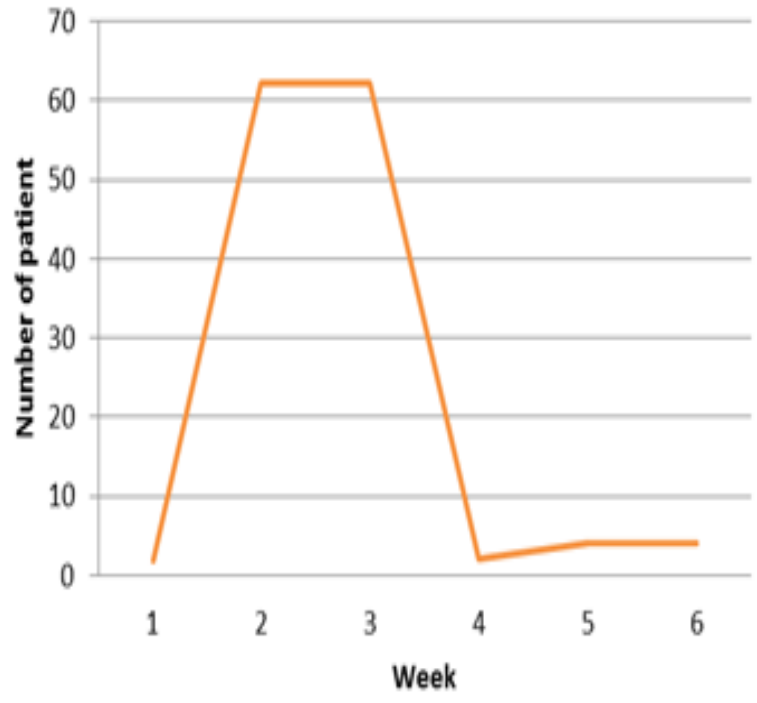

Figure 8: Queue length in ticket box

Really, in observation period, we remark a few disparities between different periods. These disparities are remark in waiting room, in registration, in waiting care room and in prescription process. To correct this case, all responsible confirm that problem can be resolving only by increase of number of medical staff and decrease administrative time in healthcare unit. Thus, nurse and doctor focus main time to care for patient.

\section{CONCLUSION}

In this paper, we presented model of medicine drugs chain in Moroccan hospital system with Colored Petri Net. We propose QueuePatient model, RegistrationPatient model and Medicine Drugs Circuit model as framework for system. With CPN Tools, we simulate these models for evaluate performance of Moroccan hospital system. We presented a making decision system in hospital which we can use to measure efficiency of system and propose some solutions. These models allow evaluating a performance of system, by fixed a means indicators: utilization resource (ticket box, doctor and register), queue delay in waiting room and in care room and lengths of queue patient. However, in our case, we focus to evaluate a total time to take care of patient and length queue patient. In next steps, we will implement these models in other hospital to compare two cases and present generic models in hospital system in Morocco.

\section{ACKNOWLEDGMENTS}

I would like to express my gratitude to all those who gave me the possibility to make this work. I want to thank the Responsible of Laboratory of Computer Systems and Renewable Energy (LISER), National Higher School of Electricity and Mechanics (ENSEM) Government of Morocco. I would like to thank Hospital in CASABLANCA for collecting a data. I want to thank all people for all their help, support, interest and valuable hints.

\section{REFERENCES}

[1] Initiation à la Medecine Sociale (IMS), (2010-2011), Moroccan's Healthcare Report, pp. 27.

[2] Info. Mgmt. Ass. (IBIMA), 2009,Vol.6, pp. 49-56.

[3] Dotoli, M., Fanti, M.P., Iacobellis, G., L. Martino, Moretti, A.M. Ukovich, W. 2010. Modeling and 
Management of a Hospital Department via Petri Nets, IEEE

[4] Huet, J.C., Paris, J.L., Gourgand, M., Kouiss. 2010. Modèle de connaissance generique du circuit du Médicament dans un hôpital, $8^{\text {th }}$ Conference Internationale de MOdelisation et SIMulation MOSIM'10

[5] Chabrol, M., Gourgand, M., Rodie, S. 2010. Modeling and evaluation of health care units "Modélisation et évaluation des unités de soins, 8e Conference Internationale de MOdelisation et SIMulation MOSIM'10

[6] Roux, O., Duvivier, D., Ramat, E., Quesnel, G., Combes, C., 2010. UML-statechart modeling tool for the VLE simulator: an application to a chronic renal dialysis unit, $8^{\text {th }}$ Conference Internationale de MOdelisation et SIMulation - MOSIM'10

[7] Moussa, M., Belkadi, K. 2009. Modélisation et Simulation de Flux Dans un Service d'Imagerie de l'HMRUO, $5^{\text {th }}$ International Conference : Sciences of Electronic, Technologies of Information and Telecommunications,March 22-26,pp.1-6.

[8] Aloui, S., Chapurlat, Penalva, V. J-M., Collomp, R., Mousnier, A., Staccini, P. J-F . 2006. ARPI ou Performance du circuit du médicament au travers du management des risques iatrogènes, Congress $\lambda \mu 15$ de l'Institut pour la Maîtrise des Risques et la Sûreté de Fonctionnement, Lille.

[9] Direction de l'Hospitalisation et de l'Organisation des Soins. 2000. L'informatisation de la chaine du médicament dans les établissements de la santé : Approche par l'analyse de la valeur: Quels projets pour quels objectifs? pp. 9

[10] Schmitt, É., Antier, D., Bernheim, C., Dufay, É., Husson, M.C., Tissot, E. 2006. Dictionnaire français de l'erreur médicamenteuse. Montry, France (ESE - Zone Industrielle)

[11] Burnel, S. 2004. Prise en charge thérapeutique du patient hospitalisé, Direction de l'Hospitalisation et de l'Organisation des Soins, Ministère de la Santé et des Solidarités, Paris, France.

[12] Hassan T. 2006. Logistique hospitalière : organisation de la chaîne logistique pharmaceutique aval et optimisation des flux de consommables et des matériels à usage unique », Ph. D. Thesis, CLAUDE BERNARD-LYON I University, France.
[13] Aloui S. 2007. Contribution à la modélisation et l'analyse du risque dans une organisation de santé au moyen d'une approche système, Ph. D. Thesis, Dept. Science and Engineering of risks activity, Ecole des Mines de Paris

[14] Baboli A., Moyaux T., Mehrabi A., Marc N. 2010. Réorganisation des réseaux de distribution d'une chaîne logistique pharmaceutique aval : Comparaison des approches centralisée et décentralisée, $8^{\text {th }}$ Conference Internationale de MOdelisation et SIMulation MOSIM'10.

[15] Sienou A. 2009. Proposition d'un cadre méthodologique pour le management intégré des risques et des processus d'entreprise, Ph.D. Thesis, Dept. Industrial Engineering, Toulouse University, France.

[16] Crespy C., Augusto V., XIE X. and BONTEMPS H. 2008. Planification et affectation des activités dans une pharmacie hospitalière par les colonies de Fourmis, $8^{\text {th }}$ Conference Internationale de MOdelisation et SIMulation - MOSIM'10.

[17] Huet J.C., Paris J.L., Gourgand M., Kouiss K., Modèle de connaissance generique du circuit du Médicament dans un hôpital, $8^{\text {th }}$ Conference Internationale de MOdelisation et SIMulation - MOSIM'10 (10 au 12 mai 2010, Hamamt, Tunisia)

[18] Huet J.C. 2011. Proposition d'une méthodologie de réingénierie pour le contrôle par le produit de systèmes manufacturiers : Application au circuit du médicament d'un hôpital, Ph.D. Thesis, Dept. Computer Sciences, BLAISE PASCAL - Clermont II University, France.

[19] Gehlot, V., Nigro, C. 2011. An Introduction to systems and simulation with Colored Petri Nets, Villanova University, Villanova, PA, USA, Proceedings Simulation Conference (WSC), Proceedings of the 2010 Winter, (Page: 104 - 118 Year of Publication: 2011 ISBN: 978-14244-9866-6)

[20] K. Jensen. 1994. An Introduction to the Theoretical Aspects of Coloured Petri Nets, Computer Science, Vol. 803, Springer-Verlag, pp. 230-272.

[21] Kristensen, L. M., Christensen S., Jensen. K.1998. The practitioner's guide to coloured Petri nets. International Journal on Software Tools for Technology Transfer, Vol.2, pp.98-132. 